\title{
Abnormal Baseline Brain Activity in Neuromyelitis Optica Patients Without Brain Lesion Detected by Resting-State Functional Magnetic Resonance Imaging
}

This article was published in the following Dove Press journal:

Neuropsychiatric Disease and Treatment

\author{
Yi Liu' ${ }^{1} *$ \\ Hua Xiong $g^{2,3, *}$ \\ Xiaojiao $\mathrm{Li}^{2,3}$ \\ Dan Zhang ${ }^{2,3}$ \\ Chao Yang ${ }^{2,3}$ \\ Jiayi $\mathrm{Yu}^{2,3}$ \\ Ruikun Liao ${ }^{2,3}$ \\ Bi Zhou ${ }^{2,3}$ \\ Xianlong Huang 2,3 \\ Zhuoyue Tang ${ }^{2,3}$ \\ 'Department of Radiology, Peking \\ University First Hospital, Beijing 100034, \\ People's Republic of China; ${ }^{2}$ Department \\ of Radiology, Chongqing General \\ Hospital, University of Chinese Academy \\ of Sciences, Chongqing 4000I4, People's \\ Republic of China; ${ }^{3}$ Molecular and \\ Functional Imaging Laboratory, \\ Chongqing General Hospital, University \\ of Chinese Academy of Sciences, \\ Chongqing 4000 I4, People's Republic of \\ China
}

*These authors contributed equally to this work
Correspondence: Zhuoyue Tang Department of Radiology, Chongqing General Hospital, University of Chinese Academy of Sciences, No. 104 Pipashan Main Street, Yuzhong District, Chongqing 4000 I4, People's Republic of China

Email zhuoyue_tang@ucas.ac.cn
Objective: To investigate the baseline brain activity in neuromyelitis optica patients without brain lesion using the regional amplitude of low-frequency fluctuation (ALFF) and fractional amplitude of low-frequency fluctuation (fALFF) as indexes.

Materials and methods: Forty-two patients of NMO with normal performance in conventional MRI and 42 healthy controls, matched in gender and age, were enrolled in this study. Resting-state functional magnetic resonance imaging (rs-fMRI) data acquired using the rs-fMRI Data Analysis Toolkit. The relationships between expanded disability states scale (EDSS) scores, abnormal baseline brain activity and disease duration were explored.

Results: The left inferior temporal, left cerebellum_4_5, bilateral superior temporal pole, left caudate, right superior temporal, left middle frontal and left superior occipital showed significantly increased ALFF in the NMO. Regions of abnormal fALFF were similar to those of ALFF except that increased fALFF were also indicated in the right cerebellum crus2, right hippocampus, left parahippocampal gyrus and left supplementary motor area. Furthermore, a significant correlation between EDSS scores and ALFF/fALFF was noted in the left inferior temporal gyrus.

Conclusion: Results confirmed the disturbances in NMO-related neural networks, which probably be related to spinal cord damage.

Keywords: neuromyelitis optica, magnetic resonance imaging, resting-state functional magnetic resonance imaging, rs-fMRI

\section{Introduction}

Neuromyelitis optica (NMO) is an inflammatory, demyelinating syndrome of the central nervous system characterized by transverse myelitis and optic neuritis. ${ }^{1,2}$ NMO is diagnosed according to the revised Wingerchuk criteria ${ }^{1}$ and has special clinical, laboratory, immunological, and pathological characteristics that distinguish it from multiple sclerosis. ${ }^{3}$ In clinical practice, visual evaluations of NMO patients mainly include ophthalmological examination, visual evoked potential (VEP), ${ }^{4}$ and magnetic resonance imaging (MRI). Conventional T1- and T2weighted (T1WI and T2WI) MRI can show the extensive transverse myelitis in patients with NMO, the relationship between the MRI findings and neurophysiologic or serological testing, but is poorly understood because both conventional MRI and clinical evaluation of spinal cord involvement are rather qualitative and subjective. 
The spinal cord is a site of predilection for NMO lesions, which often occur separately or ahead of brain lesions. NMO patients with normal brain MRI occupy the majority proportion of NMO and can be a longitudinally extensive disease that seriously influences patients' quality of life. Considering that early treatment can improve disease prognosis, early diagnosis of NMO is especially important.

Advanced MR imaging, especially functional MRI (fMRI), provides a powerful tool to noninvasively characterize the function in the brain of NMO. This technique also has the potential to further the understanding of the neuropathologic mechanisms that underlie NMO, thus helping in disease differentiation and selection of optimal therapies. Amplitude of lowfrequency fluctuation (ALFF) was used to assess the amplitude of resting-state spontaneous brain activity by calculating the square root of the power spectrum in a low-frequency range (0.01-0.08 Hz). ALFF and fractional ALFF (fALFF) reflect the increased resting-state spontaneous brain activity, which is crucial in ongoing neural circuit adaptations to the disorder caused by spinal cord damage; on the contrary, decreased ALFF and fALFF represent the decreased resting-state spontaneous brain activity, which may indicate the potential damage to the brain. ${ }^{6}$ Recent reports have demonstrated that resting-state fMRI (rs-fMRI) can assess deep gray matter damage (eg thalamus) in patients with NMO at 3.0 Tesla (T); however, those brain areas appear normal on conventional T1WI and T2WI sequences. ${ }^{7-10}$ The subjects of those studies were NMO with or without brain lesions and the alterations may be adaptations resulting from spinal cord/brain damage or potential brain changes. Whether there are abnormalities in the brain of NMO patients without brain lesion in conventional MRI is uncertain. An investigation using rs-fMRI technique focusing on brain activities of NMO patients with normal brain MRI is an interesting and essential work.

In the present study, we hypothesize that there is altered brain activity in NMO patients without brain lesion in conventional MRI. Their alterations may be considered as neural reorganization or brain plasticity in NMO patients.

\section{Materials and Methods Subjects}

This study was approved by the local Ethics Committee of The First Affiliated Hospital of Chongqing Medical University and all subjects gave written informed consent prior to participation, and this study was conducted in accordance with the Declaration of Helsinki.
Forty-two patients of NMO (24 patients with AQP4-IgG+ and 20 patients with AQP4-IgG-) without brain lesion in conventional MRI and 42 healthy controls, matched in gender, age, and right or left-handedness, were enrolled in the study. Patients were diagnosed and admitted from November 2010 to October 2015 in the Department of Neurology of the First Affiliated Hospital of Chongqing Medical University and normal volunteers were recruited from Chongqing Medical University and the surrounding communities. The subjects were all right-handed, as assessed by the Edinburgh Inventory. ${ }^{11}$ The diagnosis of NMO was according to the following criteria: (a) All patients fulfilled the revised Wingerchuk criteria: ${ }^{1}$ absolute criteria: optic neuritis and myelitis, the presence of at least two of the following three additional criteria: (1) non-diagnostic for MS at the onset. (2) MRI evidence of a spinal cord $\mathrm{T} 2$ lesion of three or more vertebral segments. (3) a serological test result positive for NMO antibodies, (b) Conventional MRI showed all patients did not detect the visible NMO brain lesions, (d) All patients did not have other neurological diseases or psychiatric problems, such as cerebrovascular disorders, hydrocephalus, an intracranial mass, psychiatric hospitalization, substance abuse, or traumatic brain injury and other myelopathy diseases. ${ }^{12}$ None of the participating patients had been treated with related medications (eg, corticosteroids and immunosuppressants) within 3 months of the MR images being obtained. As a control group, we choose 42 sex- and age-matched normal controls with no previous history of neurological dysfunction and with normal findings on neurological examination and MRI. All subjects underwent a thorough physical examination on the day of the MRI examination and were assessed the expanded disability states scale (EDSS) scores by two experienced neurologists who were unaware of the MRI results. Disease duration was calculated from symptom onset to scan date in years. In our studies, $22 \mathrm{NMO}$ were at relapses and 20 were at remission. All patients have regular MRI brain study even without relapse such every year. The main demographic and clinical characteristics of the patients are reported in Table 1.

\section{MRI Protocol}

MRI examination was performed on a 3.0 T scanner (General Electric Systems, Milwaukee, WI, USA). The matched eightchannel phased-array head coil was used. During resting-state fMRI, subjects were instructed to keep their eyes closed, to remain motionless, and not to thinks of anything in particular. Ear plugs were used to reduce scanner noise, and head motion was minimized by stabilizing the head with cushions. Conventional MRI protocols were used in all subjects during 
Table I The Main Demographic and Clinical Characteristics of Subjects

\begin{tabular}{|l|l|l|l|l|l|l|}
\hline & $\begin{array}{l}\text { No. } \\
\text { Subjects }\end{array}$ & $\begin{array}{l}\text { Mean } \\
\text { (Range) Age, } y\end{array}$ & $\begin{array}{l}\text { Sex } \\
\text { (M/F) }\end{array}$ & $\begin{array}{l}\text { Median } \\
\text { (Range) EDSS }\end{array}$ & $\begin{array}{l}\text { Median (Range) Disease } \\
\text { Duration (Month) }\end{array}$ & $\begin{array}{l}\text { Median (Range, in Spinal } \\
\text { Segments) T2 Lesion Load }\end{array}$ \\
\hline NMO group & 42 & $35.5(20-52)$ & $14 / 28$ & $3.75(1.0-7.5)$ & $20.5(2-70)$ & $3.9(0.5-5)$ \\
Control & 42 & $35.9(22-53)$ & $14 / 28$ & - & - & - \\
\hline
\end{tabular}

Abbreviation: NMO, neuromyelitis optica.

the clinical relapsing or remitting: Cranial MRI: axial T1weighed (250/2.86 ms, TR/TE), axial T1-enhanced, axial T2weighed (3600/120 ms, TR/TE), axial fluid-attenuated inversion recovery (FLAIR) (3600/120 ms, TR/TE) and sagittal FLAIR $(3600 / 120 \mathrm{~ms}$, TR/TE), the sections were obtained at $5 \mathrm{~mm}$; Spinal cord MRI: sagittal T1-weighed (500/12 ms, TR/TE), sagittal T1-enhanced, sagittal T2-weighed (2200/110 ms, TR/ TE) and axial T2-weighed (2200/110 ms, TR/TE), the sections were obtained at $4 \mathrm{~mm}$. Functional images were also used in all subjects which were recorded axially using an echo-planar imaging (EPI) sequence with the following parameters: repetition time (TR) $/$ echo time $(\mathrm{TE})=2000 / 40 \mathrm{~ms}$, flip angle $=90^{\circ}, 33$ slices, slice thickness $/$ gap $=4.0 / 0.0 \mathrm{~mm}$, voxel size $(\mathrm{mm})=$ $3.75 \times 3.75 \times 4.0$, matrix $(\mathrm{cm})=64 \times 64$, and field of view $(\mathrm{FOV})=240 \mathrm{~mm} \times 240 \mathrm{~mm}$.

\section{Data Preprocessing}

Images were preprocessed using the Statistical Parametric Mapping program (SPM12, http://www.fil.ion.ucl.ac.uk). The first 10 functional images were excluded for the signal equilibrium and participants' adaptation to scanning noise, and the subsequent EPI images were then preprocessed using the following steps: slice timing, motion correction, spatial normalization to the standard Montreal Neurological Institute (MNI) EPI template in SPM12, and resampling to $3 \mathrm{~mm} \times 3 \mathrm{~mm} \times 3 \mathrm{~mm}$, followed by spatial smoothing with a 6-mm full-width at half-maximum (FWHM) Gaussian kernel. According to the record of head motions in each fMRI run, all participants had a maximum displacement of less than $1 \mathrm{~mm}$ in the $\mathrm{x}, \mathrm{y}$, or z-plane and less than $1^{\circ}$ of angular rotation about each axis. The rs-fMRI Data Analysis Toolkit (http://restfmri.net/forum/rest v18) was then used for removing the linear trend of time courses and for temporally band-pass filtering $(0.01-0.08 \mathrm{~Hz})$.

\section{ALFF Analysis}

To determine the spontaneous brain activity pattern of each subject, we computed the ALFF and AALFF value for each voxel using the rs-fMRI Data Analysis Toolkit (http://restfmri. net/forum/rest_v18). The procedure for calculating the ALFF and $\mathrm{AALFF}$ value is similar to that used in previous studies. ${ }^{13,14}$
After preprocessing, the time series for each voxel was filtered (band-pass, $0.01-0.08 \mathrm{~Hz}$ ) to remove the effects of very-lowfrequency drift and high-frequency noise. ${ }^{15}$ Then, the filtered time series was transformed to a frequency domain with a fast Fourier transform (FFT) (parameters: taper percent $=0$, FFT length $=$ shortest), and the power spectrum was then obtained as the square root of the FFT and averaged across $0.01-0.08 \mathrm{~Hz}$ at each voxel. This averaged square root was used as the ALFF. To calculate the fALFF, a ratio of the power of each frequency at the low-frequency range $(0.01-0.08 \mathrm{~Hz})$ to that of the entire frequency range $(0-0.25 \mathrm{~Hz})$ was considered. The method of fALFF was similar to which mentioned above.

ALFF and AALFF computations and further analyses were performed within a gray matter group mask. For standardization purposes, each individual ALFF/fALFF map was divided by its own mean ALFF values within this mask. ALFF directly measures the power of the lowfrequency range while fALFF is a normalized measure. Although ALFF has somewhat higher test-retest reliability, fALFF is less susceptible to physiological artifacts. ${ }^{16}$

\section{Measurements of Spinal Cord Lesion Volume}

Visible spinal cord lesions were identified and manually extracted based on the T2-weighted image by two experienced radiologists with 12 and 10 years of neuroimaging experience and were carefully compared with fluidattenuated inversion recovery (FLAIR) images.

\section{Statistical Analysis}

A two-sample $t$-test was performed to investigate the ALFF and fALFF differences between NMO patients and normal controls, with age treated as the covariate. Voxels with a $p$-value $<0.01$ and a cluster size $>1080 \mathrm{~mm}^{3}$ (40 voxels) were considered significantly different between the two groups. The yields a corrected threshold of $p<0.05$, determined by Monte Carlo simulation using the AlphaSim program (Parameters were: FWHM $=6 \mathrm{~mm}$, with a mask of the whole-brain gray matter tissues). Correlative analysis was performed to calculate the associations between the EDSS, 
disease duration, and ALFF/fALFF in regions with significant group differences using SPSS version 17 software (SPSS, Inc., Chicago, IL).

\section{Results}

\section{Conventional MRI Features}

Conventional T1-weighed and T2-weighed images of the spinal cord in the axial and sagittal planes were examined by two experienced radiologists with 12 and 10 years of experience. NMO had hyperintense lesions in the central and dorsal areas of the spinal cord. Most of the patients had cervical or thoracic lesions (39 patients); only three patients had lumbar spine lesions. To analyze segments of spinal cord lesions, 38 patients had typically expected abnormalities (longitudinally extensive myelitis; $\geq 3$ vertebral segments spinal cord lesion) were seen on images obtained from patients (Table 2). All the lesions were lower T1 signal, higher T2 signal and higher FLAIR signal, 22 patients had Gd-enhancing lesions.

\section{ALFF and fALFF Changes}

Two-sample $t$-test was performed to assess differences between groups. As showed in Figure 1 and Table 3, after statistically controlling for the sex and age, the left inferior temporal, left cerebellum_4_5, bilateral superior temporal pole, left caudate, right superior temporal, left middle frontal and left superior occipital showed significantly increased ALFF in the NMO group than that in the controls. However, no region showed decreased ALFF values in the NMO group compared with the controls. In addition, regions of abnormal fALFF were similar to those of ALFF except that increased fALFF were also indicated in the right cerebellum crus2, right hippocampus, left parahippocampal and left supplementary motor area and there was no region showing decreased fALFF values in patients (Figure 2, Table 4).

\section{Correlations Between ALFF/fALFF Values and Clinical Data}

We examined the relationships among the EDSS, disease duration, and ALFF/fALFF in regions with significant group differences. A significant positive correlation was found between the EDSS score and ALFF value in the left inferior temporal $(r=0.805, P<0.0001)$ (Figure 3$)$. Additionally, we also found the significant correlation between the EDSS score and fALFF value in the left inferior temporal $(r=0.490, p=0.001)$ (Figure 3$)$.

\section{Discussion}

NMO is a severe demyelinating disease characterized by selective involvement of the spinal cord and optic nerves. Typically, brain MRI is normal, or in late stages, displays T2 weighted lesions in atypical sites such as the brainstem or periaqueductal areas. Baseline brain

Table 2 The Characteristics of Spinal Cord Lesions in NMO Patients

\begin{tabular}{|c|c|c|c|c|c|c|c|c|}
\hline \multirow[t]{2}{*}{ Characteristics of Lesions } & \multicolumn{4}{|c|}{ Distribution of Lesions } & \multicolumn{4}{|c|}{ Affected Segment of Spinal Cord } \\
\hline & Cervical Cord & Thoracic Cord & Lumbosacral Cord & C5-T6 & $\leq \mathbf{I}$ & I to $\leq 2$ & 2 to $\leq 3$ & $\geq 3$ \\
\hline No. lesions, $n$ & 26 & 13 & 3 & 38 & 0 & I & 3 & 38 \\
\hline Constituent ratio, $\%$ & 61.90 & 31.71 & 7.32 & 92.68 & 0 & 2.38 & 7.14 & 90.48 \\
\hline
\end{tabular}

Abbreviation: NMO, neuromyelitis optica.

Table 3 Areas Showing Significantly Changed ALFF in NMO Patients

\begin{tabular}{|l|l|l|l|l|}
\hline Anatomic Regions & Brodmann Area & Peak MNI Coordinates $(\mathbf{x}, \mathbf{y}, \mathbf{z})^{*}$ & Maximum t Score & No. of Voxels \\
\hline Left inferior temporal gyrus & 20 & $-48,-3,-36$ & 6.7679 & 144 \\
Left cerebellum_4_5 & - & $-3,-54,-6$ & 7.6619 & 265 \\
Left superior temporal pole & 38 & $-51,18,-21$ & 6.0199 & 92 \\
Right superior temporal pole & 38 & $51,12,-12$ & 6.2881 & 92 \\
Left caudate nucleus & 25 & $-3,18,-3$ & 7.4806 & 1529 \\
Right superior temporal gyrus & 22 & $45,-12,0$ & 5.0875 & 79 \\
Left middle frontal gyrus & 10 & $-36,54,21$ & 5.1504 & 76 \\
Left superior occipital gyrus & 19 & $-9,-87,45$ & 6.2761 & 95 \\
\hline
\end{tabular}

Notes: ${ }^{*} x, y$, and $z$ are the locations of the peak voxels in standard MNI coordinates. After AlphaSim correction, the cluster size was greater than 40 voxels. The threshold was set at $P<0.01$.

Abbreviation: NMO, neuromyelitis optica. 

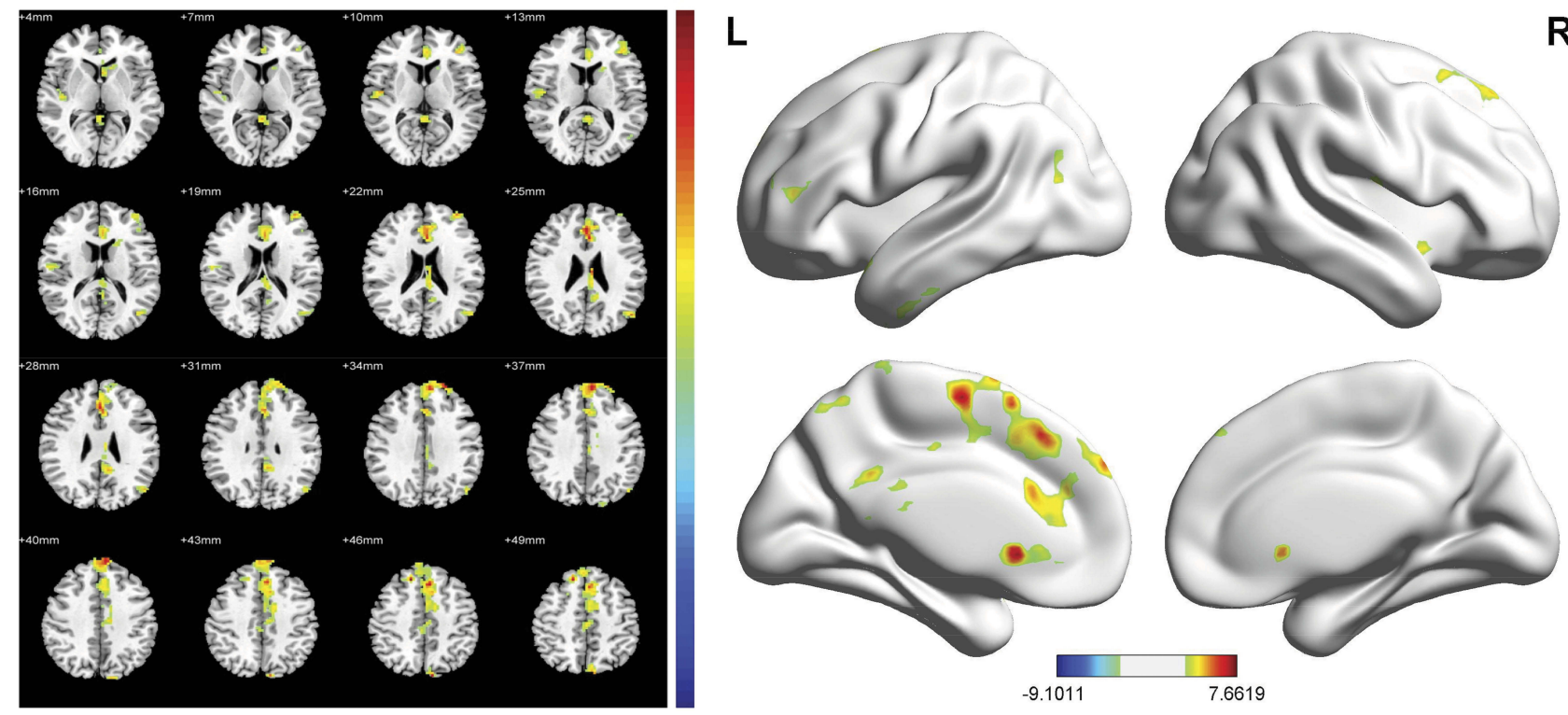

$\mathbf{R}$

Figure I The ALFF values were significantly increased between the NMO group and the control group in the left inferior temporal gyrus, left cerebellum_4_5, bilateral superior temporal pole, left caudate, right superior temporal gyrus, left middle frontal and left superior occipital gyrus. The color bar represents the $t$ values.
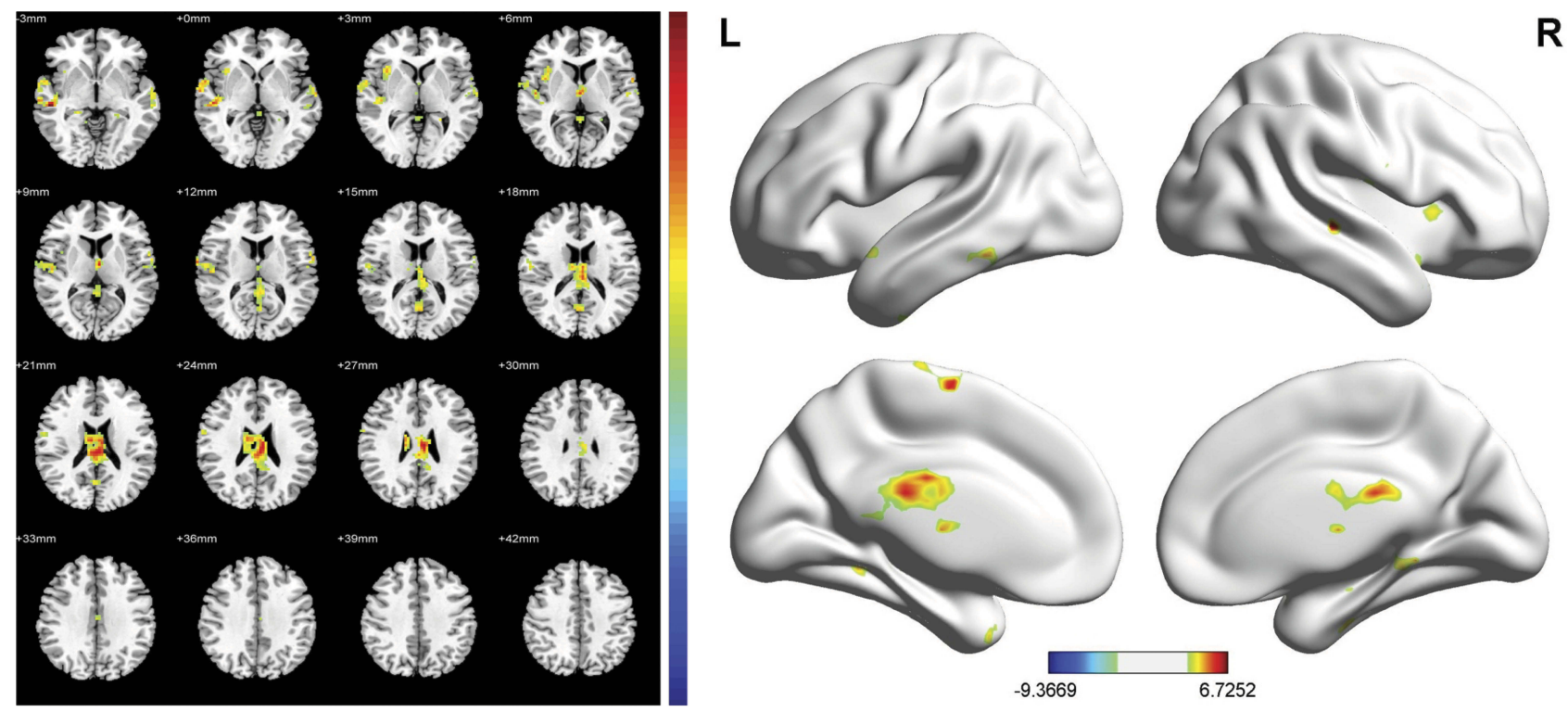

$\mathbf{R}$

Figure 2 The fALFF values were significantly increased between the NMO group and the control group in the right cerebellum crus2, right hippocampus, left parahippocampal gyrus and left supplementary motor area. The color bar represents the $t$ values.

activity is an overall reflection of functional brain damage in the resting state. Alterations in baseline brain activity represent a consequence of many brain diseases. ${ }^{17-19}$ It can reveal both function impairments and adaptations due to brain damage. Most previous fMRI investigations ${ }^{20,21}$ have revealed both functional impairments and adaptations in disease like MS, NMO, but have paid little attention to the influence of functional impairments in the brain due to spinal cord lesions. Although Liu et $\mathrm{al}^{6}$ have revealed both functional impairments and adaptations in MS without brain lesions, no study paid attention to the influence of functional impairments in the brain in NMO patients without brain lesions. To our knowledge, the current study is the first to evaluate baseline brain activity in NMO patients without brain lesions in conventional MRI.

In this study, we used ALFF and fALFF measurements to examine whole-brain spontaneous activity in the resting 
Table 4 Areas Showing Significantly Changed fALFF in NMO Patients

\begin{tabular}{|l|l|l|l|l|}
\hline Anatomic Regions & Brodmann Area & Peak MNI Coordinates (x, y, z)* & Maximum t Score & No. of Voxels \\
\hline Left inferior temporal gyrus & 20 & $-63,-42,-15$ & 6.7252 & 1196 \\
Right cerebellum crus2 & - & $54,-54,-42$ & 5.4775 & 121 \\
Right hippocampus & 28 & $36,-12,-21$ & 6.1086 & 193 \\
Left parahippocampal gyrus & 36 & $-21,-30,-18$ & 6.1638 & 90 \\
Right middle temporal gyrus & 21 & $54,-27,-3$ & 6.6243 & 257 \\
Left supplementary motor area & 6 & $-6,-6,66$ & 6.3531 & 103 \\
\hline
\end{tabular}

Notes: ${ }^{*} x, y$, and $z$ are the locations of the peak voxels in standard MNI coordinates. After AlphaSim correction, the cluster size was greater than 40 voxels. The threshold was set at $P<0.01$.

Abbreviation: NMO, neuromyelitis optica.

state in NMO patients, and our results detected abnormal neural activity in several brain areas. We detected increased ALFF values in the left inferior temporal gyrus, left cerebellum_4_5, left caudate nucleus, left middle frontal gyrus, left superior occipital gyrus, bilateral superior temporal pole and right superior temporal gyrus,
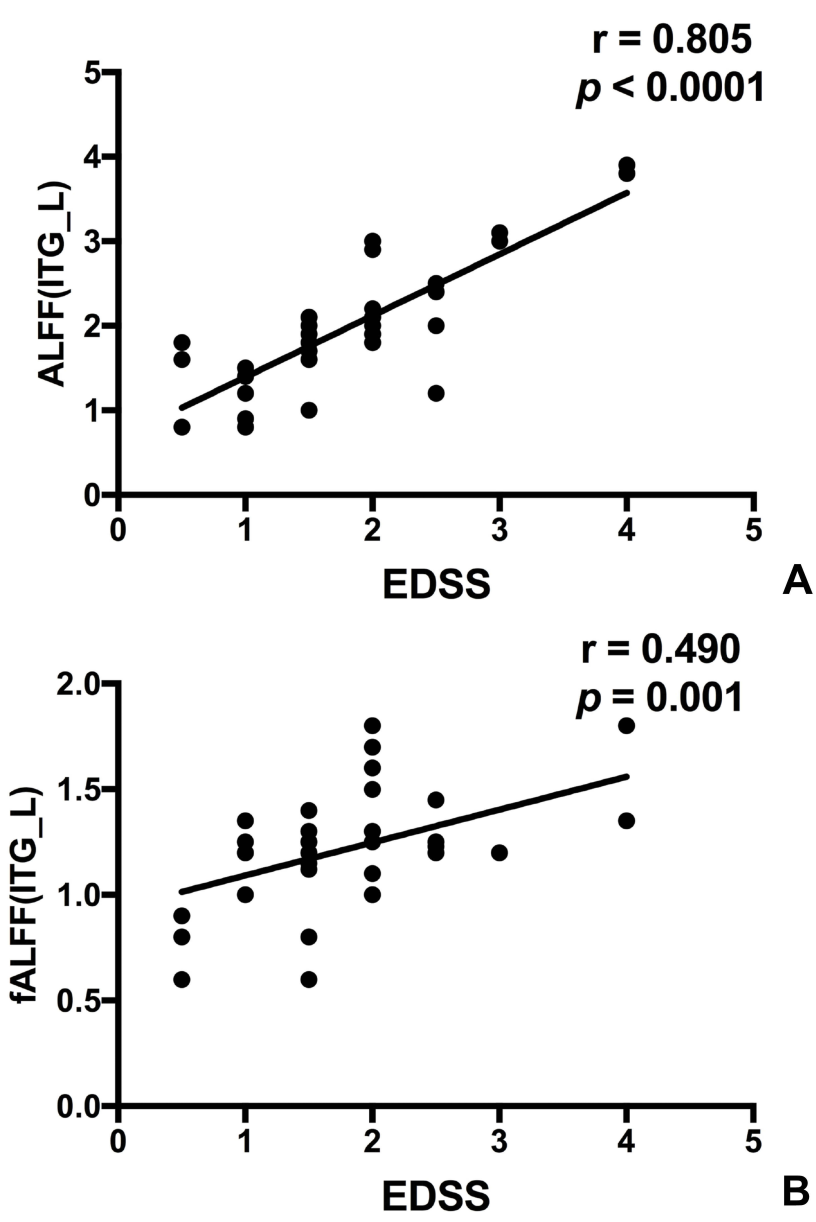

Figure 3 The correlations between the EDSS and ALFF/fALFF in left inferior temporal gyrus (ITG_L) in NMO patients. (A) The correlations between the EDSS and ALFF $(r=0.805, P<0.0001)$. (B) The correlations between the EDSS and fALFF $(r=0.490, P=0.001)$. while no decreased ALFF values were detected of NMO patients compared with the controls. The brain regions of abnormal fALFF are similar to those of ALFF except in the right cerebellum crus2, right hippocampus, left parahippocampal and left supplementary motor area. The abnormal baseline brain activity may be due to potential brain damage that could not be detected at the examined disease stage. Additionally, the ALFF and AALFF value of the left inferior temporal gyrus were positively correlated with the EDSS score.

The temporal gyrus, the main area that exhibited increased ALFF and AALFF values in the NMO group in our study, is also a widely connected brain area. The temporal is thought to influence different functions of the body, usually those that are linked to emotions or homeostatic regulation. ${ }^{22}$ The temporal gyrus has been reported to play roles in perception, motor control, self-awareness, cognitive functioning, and interpersonal experience. Abnormal activation of the temporal gyrus was observed in a variety of fMRI studies. ${ }^{23}$ Left inferior temporal gyrus (ITG), located on the lateral and inferior surface of the temporal neocortex, can be considered as a tertiary visual association cortex and the central portion of the language formulation area region, involving cognitive functions such as language, visual perception, and memory. ${ }^{24}$ Neuroimaging data support the linking of the temporal lobe to alexithymia ${ }^{25}$ Alexithymia is defined as difficulty in describing subjective feelings, and it also refers to a deficit in regulating emotions associated with somatization. ${ }^{26}$ Moreover, the left inferior temporal gyrus is consistent with the moderate positive correlation between the EDSS and the ALFF activation level, which is also with the moderate positive correlation between the EDSS and the fALFF activation level. The ALFF and fALFF increase in the temporal cortex may provide the neurobiological basis in neural circuit adaptations to the 
disorder caused by spinal cord damage. There was no negative correlation between the EDSS score and ALFF/ fALFF value in any brain area, which revealed spinal cord damage did lead to functional impairments.

The left middle frontal gyrus belongs to the default mode network (DMN). The DMN, consisting of nodes in the posterior cingulate/precuneus, bilateral middle temporal gyrus, angular gyrus and medial frontal gyrus, is most active at rest and shows reduced activity when a subject enters a task-based state involving attention or goal-directed behavior. The DMN is activated at resting state and deactivated during task activity. Husain ${ }^{27}$ pointed out that the perception of chronic tinnitus may place the subject in a task state rather than a "true resting-state". This may be due to the function of PCC: it mediates the processes of episodic memory ${ }^{28}$ and sensorimotor, as well as spatial and attentional processes. ${ }^{29}$ The left middle frontal gyrus has been widely involved in mental set maintaining $^{30}$ and responds to task difficulty. The increased ALFF and fALFF may suggest its role as substrates of functional compensation to limit the disability.

Additionally, increased caudate nucleus activity was found in our patients compared with the controls. The caudate nucleus was a key part thought to primarily be involved in the control of voluntary movement, learning and memory, particularly regarding feedback processing. The caudate is associated with the feedback of performance and learning stimulus-category associations $^{31}$ which are the main cognitive components of the symbol digit modalities test. Thus, the abnormal activation of caudate may help the explanation of the significant impairment of NMO patients in the symbol digit modalities test. A possible visual pathway to the cat caudate nucleus involving the pulvinar was also mentioned in the previous literature. ${ }^{32}$ A direct explanation for the ALFF and fALFF reduction in the left superior occipital is its role, in conjunction with the cuneus (Brodmann area 18, 19), as a visual-processing center. Therefore, we speculated that the increased ALFF in the occipital cortex is associated with the abnormal characteristic of impaired vision in NMO patients.

The right hippocampus and left parahippocampal also exhibited increased fALFF values in the NMO group. The hippocampus plays a key role in inhibiting stress responses via hypothalamic regulation, and increased exposure to adrenal glucocorticoids can accelerate hippocampal neuron loss and cognitive impairments in aging. The hippocampus contains high levels of glucocorticoid receptors, which make it more vulnerable to long-term stress than most other brain areas. ${ }^{33}$ In addition, it is noteworthy that several visual and sensory processing regions were abnormally activated in our research, which indicated a multisystem involvement in the NMO group. The left cerebellum_4_5, right cerebellum crus2 and left supplementary motor area also have different activations between the NMO patients and healthy controls. Taken together, we hypothesize that abnormalities of ALFF in the medial temporal lobe may reflect preexisting vulnerability to NMO patients.

Based on the results, the hyperactive brain areas in NMO patients were involved in cognition and visual and motor systems, consistent with previous fMRI findings in NMO and MS, ${ }^{14,34}$ which are two similar diseases. They were clinically differential diagnosis based on the 2010 revision of the McDonald criteria. ${ }^{35}$ The present research could allow for new attempts to explore the similarities and differences between NMO and MS with respect to fMRI findings. Increased ALFF and fALFF could relate to subconscious information processing. All NMO patients in our study presented increased ALFF, which may be explained by the early stage of the disease. With disease progression, the compensatory status might be replaced by the low-energy condition, further studies are needed to understand how to interpret changes in ALFF and fALFF.

There were several limitations to this study. First, the effects of physiological noise, such as cardiac and respiratory fluctuations, were not completely eliminated. Future studies should simultaneously record the cardiac and respiratory rates to address these potentially confounding variables. Second, this study is the lack of correlation between the encephalic regional changes with neuropsychological measures, such as cognitive function, sensory-motor function, working and autobiographical memory, and affective processing. In addition, the data is relatively small, which may lead to the accidental results. In the future, with greater clinical availability of advance MRI, multiple advance imaging techniques could be used together to comprehensively determine potential brain lesions of NMO patients. In addition, we can increase the amount of data and conduct the multicenter research.

\section{Conclusion}

In summary, we demonstrated functional alterations in the rs-fMRI of NMO patients without brain lesions in conventional MRI. This abnormal baseline brain activity emerged ahead of the visible brain lesions on T2WI and involves brain regions relevant to the motor and 
cognitive systems that correspond to the symptoms of NMO patients We believe these alterations may be adaptations resulting from spinal cord damage or potential brain changes that cannot be detected by conventional MRI. Results confirmed the disturbances in NMO-related neural networks, which may be potentially helpful in understanding the pathophysiology of NMO patients.

\section{Acknowledgments}

This study was supported by the medical research Key Program of the combination of Chongqing National Health Commission and Chongqing Science and Technology Bureau, China (No 2019ZDXM010); the Basic and Frontier Research Project of Chongqing, China (No cstc2016jcyjA0294); the Medical Research Key Program of the National Health and Family Planning Commission of Chongqing, China (No 20141016 and no 2016ZDXM026); and the Scientific and Technological Innovation Key Program of Chongqing General Hospital, China (No 2016ZDX M03).

\section{Disclosure}

The authors report no conflicts of interest in this work.

\section{References}

1. Wingerchuk DM, Lennon VA, Pittock SJ, Lucchinetti CF, Weinshenker BG. Revised diagnostic criteria for neuromyelitis optica. Neurology. 2006;66:1485-1489. doi:10.1212/01.wnl.0000216139.44259.74

2. Patterson SL, Goglin SE. Neuromyelitis optica. Rheum Dis Ckin North Am. 2017;43(4):579-591. doi:10.1016/j.rdc.2017.06.007

3. De Seze J, Lebrun C, Stojkovic T, Ferriby D, Chatel M, Vermersch P. Is Devic's neuromyelitis optica a separate disease? A comparative study with multiple sclerosis. Multiple Sclerosis. 2003;9:521-525. doi:10.1191/1352458503ms947oa

4. Odom JV, Bach M, Barber C, et al. Visual evoked potentials standard. Doc Ophthalmol. 2004;108:115-123. doi:10.1023/B:DOOP.00 00036790.67234 .22

5. Jeffery AR, Buncic JR. Pediatric Devic's neuromyelitis optica. J Pediatr Ophthalmol Strabismus. 1996;33:223-229.

6. Liu Y, Meng B, Zeng C, et al. Abnormal baseline brain activity in patients with multiple sclerosis with simple spinal cord involvement detected by resting-state functional magnetic resonance imaging. $J$ Comput Assist Tomogr. 2015;39(6):866-875. doi:10.1097/ RCT.0000000000000299

7. Duan Y, Liu Y, Liang P, et al. Comparison of grey matter atrophy between patients with neuromyelitis optica and multiple sclerosis: a voxel-based morphometry study. Eur J Radiol. 2012;81(2):e110-4. doi:10.1016/j.ejrad.2011.01.065

8. Zhao DD, Zhou HY, Wu QZ, et al. Diffusion tensor imaging characterization of occult brain damage in relapsing neuromyelitis optica using 3.0T magnetic resonance imaging techniques. Neuroimage. 2012;59(4):3173-3177. doi:10.1016/j.neuroimage.2011.11.022
9. Chanson JB, Lamy J, Rousseau F, et al. White matter volume is decreased in the brain of patients with neuromyelitis optica. Eur J Neurol. 2013;20 (2):361-367. doi:10.1111/j.1468-1331.2012.03867.x

10. Liu Y, Duan Y, Huang J, et al. Multiple quantitative MR Imaging of the thalamus in multiple sclerosis and neuromyelitis optica. Radiology. 2015;3:784-792. doi:10.1148/radiol.2015142786

11. Oldfield RC. The assessment and analysis of handedness: the Edinburgh inventory. Neuropsychologia. 1971;9(1):97-113. doi:10.1016/0028-3932(71)90067-4

12. Chanson JB, Alame M, Collongues N, et al. Evaluation of clinical interest of anti-aquaporin-4 autoantibody followup in neuromyelitis optica. Clin Dev Immunol. 2013;146219. doi: 10.1155/2013/146219

13. Zang YF, He Y, Zhu CZ, et al. Altered baseline brain activity in children with ADHD revealed by resting-state functional MRI. Brain Dev. 2007;29(2):83-91. doi:10.1016/j.braindev.2006.07.002

14. Liu Y, Liang P, Duan Y, et al. Abnormal baseline brain activity in patients with neuromyelitis optica: a resting-state fMRI study. Eur J Radiol. 2011;80(2):407-411. doi:10.1016/j.ejrad.2010.05.002

15. Biswal B, Yetkin FZ, Haughton VM, Hyde JS. Functional connectivity in the motor cortex of resting human brain using echo-planar MRI. Magn Reson Med. 1995;34(4):537-541. doi:10.1002/(ISSN)1522-2594

16. Zuo XN, Di Martino A, Kelly C, et al. The oscillating brain: complex and reliable. Neuroimage. 2010;49:1432-1445. doi:10.1016/j. neuroimage.2009.09.037

17. Wen X, Wu X, Liu J, Yao L. Abnormal baseline brain activity in nondepressed Parkinson's disease and depressed Parkinson's disease: a resting-state functional magnetic resonance imaging study. PLoS One. 2013;8(5):63691. doi:10.1371/journal.pone.0063691

18. Khadka S, Meda SA, Stevens MC, et al. Is aberrant functional connectivity a psychosis endophenotype? A resting state functional magnetic resonance imaging study. Biol Psychiatry. 2013;74 (6):458-466. doi:10.1016/j.biopsych.2013.04.024

19. Feng T, Feng P, Chen Z. Altered resting-state brain activity at functional MRI during automatic memory consolidation of fear conditioning. Brain Res. 2013;1523:59-67. doi:10.1016/j. brainres.2013.05.039

20. Rueda-Lopes FC, Hygino da Cruz LC Jr, Doring TM, Gasparetto EL. Diffusion-weighted imaging and demyelinating diseases: new aspects of an old advanced sequence. AJR Am J Roentgenol. 2014;202:W34W42. doi:10.2214/AJR.13.11400

21. Rocca MA, Absinta M, Moiola L, et al. Functional and structural connectivity of the motor network in pediatric and adult-onset relapsing-remitting multiple sclerosis. Radiology. 2010;254 (2):541-550. doi:10.1148/radiol.09090463

22. Xu K, Liu H, Li H, et al. Amplitude of low-frequency fluctuations in bipolar disorder: a resting state fMRI study. J Affect Dis. 2014;152-154:237-242. doi:10.1016/j.jad.2013.09.017

23. Wegner C, Filippi M, Korteweg T, et al. Relating functional changes during hand movement to clinical parameters in patients with multiple sclerosis in a multi-centre fMRI study. Eur J Neurol. 2008;15 (2):113-122. doi:10.1111/j.1468-1331.2007.02027.x

24. Dien J, Brian ES, Molfese DL, Gold BT. Combined ERP/fMRI evidence for early word recognition effects in the posterior inferior temporal gyrus. Cortex. 2013;49:2307-2321. doi:10.1016/j. cortex.2013.03.008

25. Aust S, Alkan Hartwig E, Koelsch S, Heekeren HK, Heuser I, Bajbouj M. How emotional abilities modulate the influence of early life stress on hippocampal functioning. Soc Cogn Affect Neurosci. 2014;9:1038-1045. doi:10.1093/scan/nst078

26. Bailey PE, Henry JD. Alexithymia, somatization and negative affect in a community sample. Psychiatry Res. 2007;150:1-20. doi:10.1016/ j.psychres.2006.05.024

27. Mantini D, Perrucci MG, Del Gratta C, Romani GL, Corbetta M. Electrophysiological signatures of resting state networks in the human brain. Proc Natl Acad Sci USA. 2007;104(32):13170-13175. doi:10.1073/pnas.0700668104 
28. Husain FT, Schmidt SA. Using resting state functional connectivity to unravel networks of tinnitus. Hear Res. 2014;307:153-162. doi:10.1016/j.heares.2013.07.010

29. De Ridder D, Vanneste S, Congedo M. The distressed brain: a group blind source separation analysis on tinnitus. PLoS One. 2011;6(10): e24273. doi:10.1371/journal.pone.0024273

30. Sorg C, Riedl V, Muhlau M, et al. Selective changes of resting-state networks in individuals at risk for Alzheimer's disease. Proc Natl Acad Sci USA. 2007;104(47):18760-18765. doi:10.1073/pnas.07088 03104

31. Seger CA, Cincotta CM. The roles of the caudate nucleus in human classification learning. J Neurosci. 2005;25:2941-2951. doi:10.1523/ JNEUROSCI.3401-04.2005
32. Cincotta CM, Seger CA. Dissociation between striatal regions while learning to categorize via feedback and via observation. J Cogn Neurosci. 2007;19:249-265. doi:10.1162/jocn.2007.19.2.249

33. Joels M. Functional actions of corticosteroids in the hippocampus. Eur J Pharmacol. 2008;583:312-321. doi:10.1016/j. ejphar.2007.11.064

34. Liu Y, Liang P, Duan Y, et al. Brain plasticity in relapsing-remitting multiple sclerosis: evidence from resting-state fMRI. J Neurol Sci. 2011;304(1-2):127-131. doi:10.1016/j.jns.2011.01.023

35. Selchen D, Bhan V, Blevins G, et al. MS, MRI, and the 2010 McDonald criteria: a Canadian expert commentary. Neurology. 2012;79(23):1-15. doi:10.1212/WNL.0b013e318277d144

\section{Publish your work in this journal}

Neuropsychiatric Disease and Treatment is an international, peerreviewed journal of clinical therapeutics and pharmacology focusing on concise rapid reporting of clinical or pre-clinical studies on a range of neuropsychiatric and neurological disorders. This journal is indexed on PubMed Central, the 'PsycINFO' database and CAS, and is the official journal of The International Neuropsychiatric Association (INA). The manuscript management system is completely online and includes a very quick and fair peer-review system, which is all easy to use. Visit http://www.dovepress.com/testimonials.php to read real quotes from published authors. 